\title{
Robust and efficient designs for the Michaelis-Menten model
}

\author{
Holger Dette \\ Ruhr-Universität Bochum \\ Fakultät für Mathematik \\ 44780 Bochum \\ Germany
}

email: holger.dette@ruhr-uni-bochum.de

FAX: +492347094559

\author{
Stefanie Biedermann \\ Ruhr-Universität Bochum \\ Fakultät für Mathematik \\ 44780 Bochum \\ Germany
}

email: stefanie.biedermann@ruhr-uni-bochum.de

April 3, 2002

\begin{abstract}
For the Michaelis-Menten model we determine designs which maximize the minimum of the $D$-efficiencies over a certain interval for the non-linear parameter. The best two point designs can be found explicitly and a characterization is given, when these designs are optimal within the class of all designs. In most cases of practical interest the determined designs are highly efficient and robust with respect to misspecification of the non-linear parameter. The results are illustrated and applied in an example of hormone-receptor assay.
\end{abstract}

Keywords and Phrases: Michaelis-Menten model, robust optimal design, local D-optimality, receptor assay

\section{Introduction}

The rectangular hyperbola has been used to describe saturation functions for numerous physical and biological phenomena [see Cressie and Keightley (1979), Johansen (1984), Beverton and 
Holt (1957), Cornish-Browden (1979), Hay, Meznarich, DiGiacomo, Hirst, Zerbe (1988)]. The form encountered most often in biology is the familiar Michaelis-Menten enzyme kinetic function

$$
y=\frac{a x}{b+x} ; \quad x \in\left[0, x_{0}\right],
$$

where $y$ is the reaction velocity, $a$ the maximum velocity of this reaction, $x$ the concentration of a substrate and $b$ the half-saturation constant, the value of $x$, where $y$ is half-maximal. Several methods have been proposed in the literature to estimate the parameters $a, b$ [see e.g. Bliss and James (1966), Glick, Landman and Roufogalis (1979), Zivin and Waud (1982), Currie (1982) or Raaijmakers (1987)]. Most of these are based on transformations of the equation (1.1) to a linear plot and an application of ordinary linear regression techniques. Recently Raaijmaakers (1987) gave some arguments in favor of the application of maximum likelihood techniques for the estimation of the parameters in the Michaelis-Menten model.

The problem of designing experiments for the Michaelis-Menten model has also found considerable attention in the literature [see e.g. Duggleby (1979), Dunn (1988), Boer, Rasch and Hendrix (2000) or Dette and Wong (1999)]. However, optimal designs are difficult to implement in practice, because in non-linear models the (locally) optimal designs depend on the unknown parameters [see Chernoff (1953) or Silvey (1980)] and usually are not robust with respect to their misspecification. For this reason, Song and Wong (1998) proposed Bayesian optimal designs for the Michael-Menten model and studied some of their properties. In this paper we study a different approach, which is based on the minimax concept [see Müller (1995), Haines (1995), Dette (1997) or Imhof (2001)]. For the sake of transparency, we concentrate on maximum likelihood estimation and the $D$-optimality criterion, which means that an (locally) optimal design maximizes the determinant of the Fisher information matrix. Following Dette (1997) we propose as an optimality criterion the maximization of the minimum $D$-efficiency (with respect to the locally $D$-optimal design) taken over a certain range for the parameters. This criterion is motivated by the fact that in many cases the experimenter is able to specify a certain range for the non-linear parameters [see e.g. Cressie and Keightley (1982)], but has no preference for specific values, which would allow the specification of a prior distribution for a Bayesian approach. The optimality criterion is carefully defined in Section 2, which also discusses a useful reparametrization of the model. In Section 3 we determine the standardized maximin optimal two point designs and give an explicit characterization, when these designs are optimal within the class of all designs. It is demonstrated that in most cases the optimal designs are supported on two points and have equal masses at these points. Moreover, in the cases where the standardized maximin optimal designs are supported at more than two points it is indicated that the best two point designs are rather efficient. Section 4 contains a brief discussion of designing experiments for the Michaelis-Menten model in the case of heteroscedasticity and gives the (standardized) maximin designs in the case, where the variance is a quadratic function of the mean [see Dunn (1988)]. Finally, some of the more technical arguments are deferred to an appendix. 


\section{Standardized maximin optimal designs}

Recalling the definition of the model (1.1) we introduce a slightly different parametrization, that is

$$
a=\alpha x_{0}, \quad b=\beta x_{0},
$$

and assume that $Y$ follows an exponential distribution with mean

$$
E[Y \mid x]=\frac{\alpha x_{0} x}{\beta x_{0}+x}, \quad x \in\left[0, x_{0}\right]
$$

and constant variance. The Fisher information for the parameters $(\alpha, \beta)$ at a point $x$ is then given by

$$
I(x, \alpha, \beta)=x_{0}^{2} \frac{\left(x / x_{0}\right)^{2}}{\left(\beta+x / x_{0}\right)^{2}}\left(\begin{array}{cc}
1 & -\frac{\alpha}{\beta+x / x_{0}} \\
-\frac{\alpha}{\beta+x / x_{0}} & \frac{\alpha^{2}}{\left(\beta+x / x_{0}\right)^{2}}
\end{array}\right) .
$$

A design is a probability measure $\xi$ on the interval $\left[0, x_{0}\right]$ with finite support, where the support points correspond to the location of the observations and the weights give the relative proportions of total observations to be taken at the corresponding points. The Fisher information matrix of a design $\xi$ is defined as

$$
M(\xi, \alpha, \beta)=\int_{0}^{x_{0}} I(x, \alpha, \beta) d \xi(x)
$$

and a locally $D$-optimal design $\xi_{\alpha, \beta}^{*}$ maximizes the determinant of the matrix $M(\xi, \alpha, \beta)$. Observing the structure of the Fisher information $I(x, \alpha, \beta)$ defined in $(2.3)$ and the fact that we are discussing the determinant criterion, it is easy to see that the factor $\alpha$ has no effect on the maximization of the determinant $|M(\xi, \alpha, \beta)|$, and we may assume without loss of generality $\alpha=1$. For the sake of a transparent notation we introduce

$$
M(\xi, \beta)=M(\xi, 1, \beta) .
$$

Similarly, it is easy to see that we can restrict ourselves to the case $x_{0}=1$ and that designs on the general interval $\left[0, x_{0}\right]$ are simply obtained by rescaling the corresponding measures on $[0,1]$ with the factor $x_{0}$. For example the locally $D$-optimal design on the interval $[0,1]$ is given by the measure

$$
\xi_{\beta}^{*}=\left(\begin{array}{cc}
\frac{\beta}{2 \beta+1} & 1 \\
\frac{1}{2} & \frac{1}{2}
\end{array}\right)
$$


(here the first row represents the support points and the second row the corresponding weights) and the locally $D$-optimal design on the interval $\left[0, x_{0}\right]$ has equal masses at the points $\beta x_{0} /(2 \beta+$ 1 ) and $x_{0}$ [see Rasch (1990)]. The value of the determinant of the locally $D$-optimal design can easily be calculated as

$$
\left|M\left(\xi_{\beta}^{*}, \beta\right)\right|=\frac{1}{64 \beta^{2}(1+\beta)^{6}} .
$$

Note that the optimal design depends sensitively on the unknown parameter $\beta$ and some prior knowledge regarding this parameter is necessary to implement this design in practice. However, precise knowledge of this parameter is rarely available in practical problems and for this reason locally optimal designs are not too widely used in applications. Nevertheless, in many situations a certain range for the parameter $\beta$ can be specified by the experimenter [see for example Cressie and Keightley (1981), p.237, where a specific range for the dissociation constant $\beta$ for the receptor-estradiol interaction is given] and a maximin approach might be appropriate which maximizes the minimum of the determinants $|M(\xi, \beta)|$ taken over a certain interval, say $\left[\beta_{0}, \beta_{1}\right]$, for the parameter $\beta$ with respect to the choice of the design $\xi$. It was pointed out by Dette (1997) in a more general context that the values of the determinants $|M(\xi, \beta)|$ at different points $\beta$ might be of rather different size and for this reason not comparable. In fact it can be shown by an application of Hölder's inequality that $|M(\xi, \beta)|$ is decreasing with increasing value of $\beta$ (independently of the design $\xi$ ) and consequently the design maximizing the minimum of the determinants is in fact locally $D$-optimal for the smallest possible parameter $\beta_{0}$, for which the minimum is computed. Obviously this design is inefficient for large values in the interval $\left[\beta_{0}, \beta_{1}\right]$. A more reasonable criterion, which takes different sizes of the determinants into account, is the standardized maximin D-optimality criterion [see Dette (1997)]

$$
\begin{aligned}
\Phi(\xi) & =\min \left\{\frac{|M(\xi, \beta)|}{\sup _{\eta}|M(\eta, \beta)|} \mid \beta \in\left[\beta_{0}, \beta_{1}\right]\right\} \\
& =\min \left\{\frac{|M(\xi, \beta)|}{\left|M\left(\xi_{\beta}^{*}, \beta\right)\right|} \mid \beta \in\left[\beta_{0}, \beta_{1}\right]\right\} .
\end{aligned}
$$

This criterion seeks for a design maximizing the worst $D$-efficiency

$$
\operatorname{eff}_{\beta}(\xi)=\left(\frac{|M(\xi, \beta)|}{\left|M\left(\xi_{\beta}^{*}, \beta\right)\right|}\right)^{1 / 2}
$$

over the interval $\left[\beta_{0}, \beta_{1}\right]$. Following Dette (1997) a design $\xi^{*}$ maximizing the criterion $\Phi$ in $(2.6)$ is called standardized maximin $D$-optimal design. The following result characterizes the design $\xi^{*}$ and can be proved by standard methods [see e.g. Wong (1992) or Pukelsheim (1993)]. 
Lemma 2.1. A design $\xi^{*}$ is standardized maximin D-optimal if and only if there exists a prior $\pi$ supported on the set

$$
\mathcal{N}\left(\xi^{*}\right)=\left\{\beta \in\left[\beta_{0}, \beta_{1}\right] \mid \Phi\left(\xi^{*}\right)=\left[\operatorname{eff}_{\beta}\left(\xi^{*}\right)\right]^{2}\right\}
$$

such that the inequality

$$
\int_{\left[\beta_{0}, \beta_{1}\right]} f^{T}(x, \beta) M^{-1}\left(\xi^{*}, \beta\right) f(x, \beta) \pi(d \beta) \leq 2
$$

holds for all $x \in[0,1]$, where

$$
f^{T}(x, \beta)=\frac{x}{\beta+x}\left(1,-\frac{1}{\beta+x}\right) .
$$

Moreover, there is equality in (2.10) for all support points of the design $\xi^{*}$.

Although Lemma 2.1 is attractive from a theoretical point of view, its practical impact is limited, because it does not answer the question how to choose the prior $\pi$ in (2.10). For this reason, standardized maximin optimal designs have only been found in rare circumstances [see Müller (1995), or Imhof (2001)]. In all of these cases the authors concentrate on the optimization in the subclass of minimum supported designs. To be precise, we call a design with $k$ support points standardized maximin $D$-optimal $k$-point design, if it maximizes the function $\Phi$ defined in (2.7) in the class of all designs with at most $k$ support points. Our main result of this section gives the standardized maximin $D$-optimal two point design for the Michaelis-Menten model. The proof is deferred to the appendix.

Theorem 2.2. The standardized maximin D-optimal two point design $\xi_{z^{*}}$ for the MichaelisMenten model on the interval $[0,1]$ has equal masses at the points 1 and

$$
z^{*}=z^{*}\left(\beta_{0}, \beta_{1}\right)=\frac{\beta_{1} \sqrt{\beta_{0}\left(1+\beta_{0}\right)}-\beta_{0} \sqrt{\beta_{1}\left(1+\beta_{1}\right)}}{\sqrt{\beta_{1}\left(1+\beta_{1}\right)}-\sqrt{\beta_{0}\left(1+\beta_{0}\right)}} .
$$

Moreover, for this design the set $\mathcal{N}\left(\xi_{z^{*}}\right)$ defined in (2.9) is given by

$$
\mathcal{N}\left(\xi_{z^{*}}\right)=\left\{\beta_{0}, \beta_{1}\right\} \text {. }
$$

The question if and when the design $\xi_{z^{*}}$ coincides with the standardized maximin $D$-optimal design within the class of all designs will be deferred to the following section. Roughly speaking this statement is correct, whenever

$$
\frac{\beta_{0}}{\beta_{1}} \geq \frac{7}{36}
$$


but the lower bound can be improved (decreased) with increasing value of $\beta_{1}$. We will conclude this section with an example illustrating the transformation introduced in (2.1) and the application of Theorem 2.2 for finding the best two point designs.

Example 2.3. For the analysis of data from hormone-receptor assays the relation between the response and explanatory variable is usually modelled by the Michaelis-Menten equation [see Cressie and Keightley (1981)]. In the specific example considered by these authors the range for the variable $x$ is the interval $\left[0, x_{0}\right]=[0,2000]$. We consider three possible ranges for the interval for the parameter $b$ in the model (1.1), namely

$$
\begin{aligned}
& B_{A}=[100,2000] \\
& B_{B}=[100,1000] \\
& B_{C}=[100,500]
\end{aligned}
$$

Using the transformation (2.1) this gives for the $\beta$-scale the intervals $[0.05,1],[0.05,0.5]$ and $[0.05,0.25]$, respectively. The standardized maximin $D$-optimal two point designs are now obtained from Theorem 2.2 and the transformation $x \rightarrow 2000 x$, which gives

$$
\begin{aligned}
\xi_{A} & =\left(\begin{array}{cc}
267.35 & 2000 \\
\frac{1}{2} & \frac{1}{2}
\end{array}\right) \\
\xi_{B} & =\left(\begin{array}{cc}
223.78 & 2000 \\
\frac{1}{2} & \frac{1}{2}
\end{array}\right) \\
\xi_{C} & =\left(\begin{array}{cc}
177.83 & 2000 \\
\frac{1}{2} & \frac{1}{2}
\end{array}\right) .
\end{aligned}
$$

It follows from the proof of Theorem 2.2 in the Appendix that the worst $D$-efficiency of the standardized maximin $D$-optimal two point design is given by

$$
\min _{\beta \in\left[\beta_{0}, \beta_{1}\right]} \operatorname{eff}\left(\xi_{z^{*}}\right)=\frac{4 \beta_{0}\left(1+\beta_{0}\right) z^{*}\left(1-z^{*}\right)}{\left(z^{*}+\beta_{0}\right)^{2}}=\left[u\left(z^{*}, \beta_{0}\right)\right]^{1 / 2} .
$$

Figures $1-3$ show the $D$-efficiencies (with respect to the locally $D$-optimal design $\xi_{\beta}^{*}$ ) of the designs $\xi_{A}, \xi_{B}$ and $\xi_{C}$ given in $(2.13)$ - (2.15). We observe that for a broad range of the intervals $B_{A}, B_{B}$ and $B_{C}$ the standardized maximin $D$-optimal two point design has at least local $D$-efficiency $80 \%$. For example, if we used the design $\xi_{B}$ and the true parameter in the model (1.1) would be $b=600$, then the design $\xi_{B}$ has efficiency $90 \%$ compared to the locally $D$-optimal design, which assumes precise knowledge of $b$. 


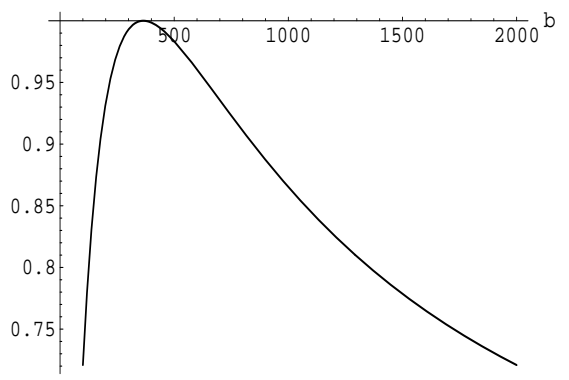

Figure 1: $D$-efficiencies of the standardized maximin $D$-optimal two point design $\xi_{A}$, where the minimum is taken over the range $[100,2000]$

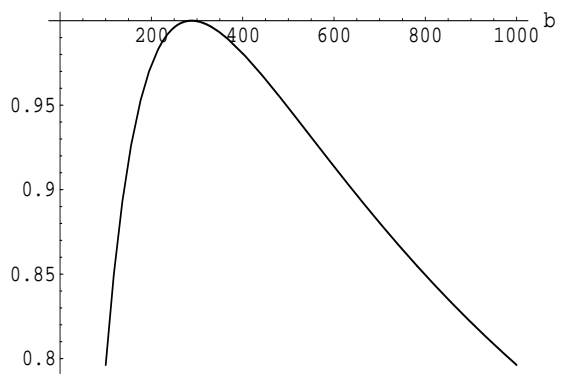

Figure 2: $D$-efficiencies of the standardized maximin $D$-optimal two point design $\xi_{B}$, where the minimum is taken over the range $[100,1000]$

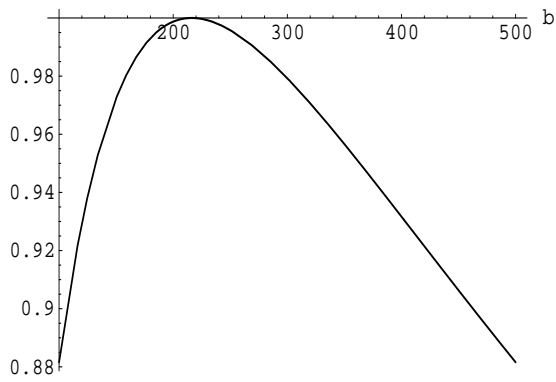

Figure 3: $D$-efficiencies of the standardized maximin $D$-optimal two point design $\xi_{C}$, where the minimum is taken over the range $[100,500]$ 


\section{Global optimality and efficiency considerations}

The optimality of the determined best two point designs can be checked with the equivalence theorem given in Lemma 2.1. The following result determines the prior $\pi$, which is the main ingredient in Lemma 2.1. The proof is deferred to the appendix.

Lemma 3.1. Let $\xi_{z^{*}}$ denote the standardized maximin D-optimal two point design given in Theorem 2.2, then $\mathcal{N}\left(\xi_{z^{*}}\right)=\left\{\beta_{0}, \beta_{1}\right\}$ and the only prior on the set $\mathcal{N}\left(\xi_{z^{*}}\right)$, for which the checking condition (2.10) is eventually satisfied, is the uniform distribution on $\mathcal{N}\left(\xi_{z^{*}}\right)$.

Note that it is not guaranteed that the design $\xi_{z^{*}}$ of Theorem 2.2 and the uniform prior on the set $\left\{\beta_{0}, \beta_{1}\right\}$ satisfy the inequality (2.10), but the only prior for which this could be possible is the uniform prior. Consequently, we can use Lemma 2.1 with the uniform prior in order to check the optimality of the best two point design $\xi_{z^{*}}$ given in Theorem 2.2. A straightforward calculation shows that the inequality (2.10) holds for all $x \in[0,1]$, if and only if the function

$$
L\left(\xi_{z^{*}}\right)=\frac{2}{\max _{x \in[0,1]} \int_{\beta_{0}}^{\beta_{1}} f^{T}(x, \beta) M^{-1}\left(\xi_{z^{*}}, \beta\right) f(x, \beta) \pi(d \beta)}
$$

satisfies the equation

$$
L\left(\xi_{z^{*}}\right)=1
$$

Moreover, it was shown by Dette (1996) that the function $L$ proves a lower boundary for the $\Phi$-efficiency of a design with respect to the standardized maximin optimality criterion defined in $(2.7)$, that is

$$
\operatorname{eff}_{\Phi}(\xi)=\frac{\Phi(\xi)}{\max _{\eta} \Phi(\eta)} \geq L(\xi)
$$

for all designs $\xi$. For the best two point design $\xi_{z^{*}}$ and the uniform prior we obtain by a straightforward calculation that the inequality (2.10) is equivalent to

$$
g\left(x, \beta_{0}, \beta_{1}\right) \leq 2 \quad \forall x \in[0,1]
$$

and the function $L$ is given by

$$
L\left(\xi_{z^{*}}\right)=\frac{2}{\max _{x \in[0,1]} g\left(x, \beta_{0}, \beta_{1}\right)},
$$


where the function $g$ is defined by

$$
\begin{aligned}
g\left(x, \beta_{0}, \beta_{1}\right)= & \sum_{i=0}^{1} \frac{\left(1+\beta_{i}\right)^{4}\left(z^{*}+\beta_{i}\right)^{4} x^{2}}{\left(z^{*}\right)^{2}\left(1-z^{*}\right)^{2}\left(x+\beta_{i}\right)^{2}}\left\{\frac{\left(z^{*}\right)^{2}}{\left(z^{*}+\beta_{i}\right)^{4}}+\frac{1}{\left(1+\beta_{i}\right)^{4}}\right. \\
& \left.-\frac{2}{x+\beta_{i}}\left(\frac{\left(z^{*}\right)^{2}}{\left(z^{*}+\beta_{i}\right)^{3}}+\frac{1}{\left(1+\beta_{i}\right)^{3}}\right)+\frac{1}{\left(x+\beta_{i}\right)^{2}}\left(\frac{\left(z^{*}\right)^{2}}{\left(z^{*}+\beta_{i}\right)^{2}}+\frac{1}{\left(1+\beta_{i}\right)^{2}}\right)\right\} .
\end{aligned}
$$

Example 3.2. Recall the situation of Example 2.3. The condition (3.4) for the best two point designs in (2.13) - (2.15) is illustrated in Figure 4, where the designs have been rescaled to the interval $[0,1]$. From the inequality (3.4) it follows that the design $\xi_{C}$ is the standardized maximin $D$-optimal design in the class of all designs, and that this conclusion is not correct for the designs $\xi_{A}$ and $\xi_{B}$ (see Figure 4). However, we obtain from (3.3) and (3.5) the lower bounds $80.24 \%$ and $93.8 \%$ for the $\Phi$-efficiencies of the designs $\xi_{A}$ and $\xi_{B}$, respectively. Thus one cannot expect a too substantial improvement by determining the standardized maximin $D$-optimal design in the class of all designs (note that $L\left(\xi_{z^{*}}\right)$ provides only a lower bound for the $\Phi$-efficiency and the true $\Phi$-efficiency could be in fact substantially larger).

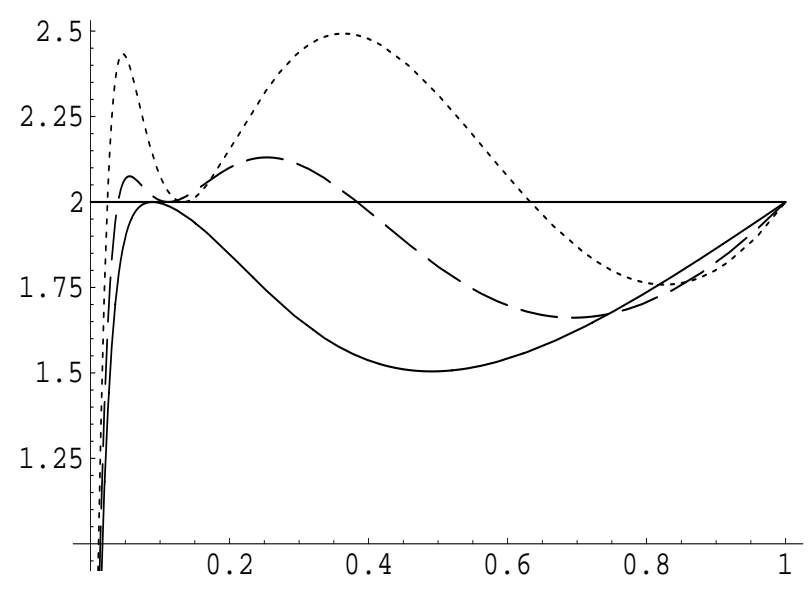

Figure 4: The function $g$ defined in (3.6) for the designs $\xi_{A}$ (dotted line), $\xi_{B}$ (dashed line) and $\xi_{C}$. The design is standardized maximin $D$-optimal if and only if the curve stays below the line $x=2$. Twice the inverse of the maximal excess gives a lower bound for the $\Phi$-efficiency [see inequality (3.3)]

For the following discussion we use a slightly different parametrization of the interval $\left[\beta_{0}, \beta_{1}\right]$. 
Let $c \in(0,1]$ be defined by

$$
\beta_{0}=c \beta_{1}
$$

and consider the function

$$
h\left(c, \beta_{1}\right)=\max _{x \in[0,1]} g\left(x, c \beta_{1}, \beta_{1}\right),
$$

where the function $g$ is given in (3.6). By Lemma 2.1 and the discussion preceding Example 3.2 it follows that the best two point design is in fact standardized maximin $D$-optimal within the class of all measures if and only if $h\left(c, \beta_{1}\right)=2$. For example, for the choice $c=1$, the interval $\left[\beta_{0}, \beta_{1}\right]$ collapses to the point $\beta_{1}$ and the standardized maximin $D$-optimality criterion reduces to the local $D$-optimality criterion. We trivially obtain $h\left(1, \beta_{1}\right)=2$, because the locally $D$-optimal design is in fact supported on two points. We are now interested in the bound

$$
k\left(\beta_{1}\right)=\inf \left\{c \in(0,1] \mid h\left(u, \beta_{1}\right)=2 \quad \forall u \geq c\right\} .
$$

In other words $k\left(\beta_{1}\right)$ is the smallest number in the interval $(0,1]$, such that for any value $c>k\left(\beta_{1}\right)$ the best two point design of Theorem 2.2 is actually the standardized maximin $D$-optimal design in the class of all models (where the minimum is taken over the interval $\left.\left[c \beta_{1}, \beta_{1}\right]\right)$. Although an analytical derivation of this function seems to be intractable, our numerical calculations show that it is in fact inverse linear. More precisely, our numerical results indicate that $k\left(\beta_{1}\right)$ is determined by the relation

$$
k\left(\beta_{1}\right)=\frac{1}{4.14307231 \beta_{1}+5.14307231} .
$$

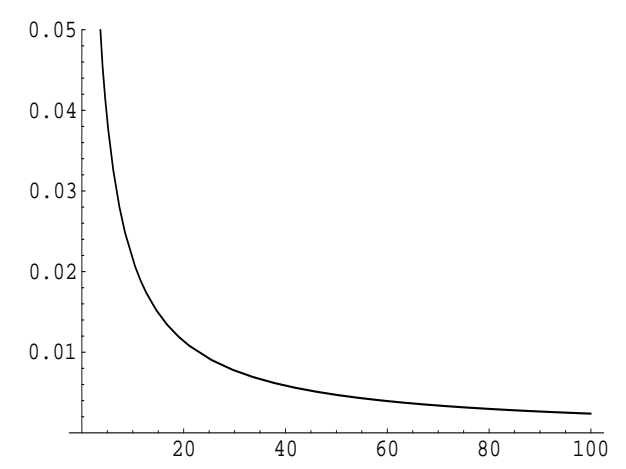

Figure 5: The function $k$ defined in (3.7) for $\beta_{1} \in(0,100]$

The function $k$ is depicted in Figure 5 for a broad range of $\beta_{1}$-values. Moreover, our numerical study shows that for all values smaller than $k\left(\beta_{1}\right)$ the best two point design is not optimal within the class of all designs. In other words: 
Remark 3.3. The standardized maximin D-optimal two point design given in Theorem 2.2 is standardized maximin D-optimal within the class of all designs, if and only if

$$
c=\frac{\beta_{0}}{\beta_{1}} \geq k\left(\beta_{1}\right)
$$

where the function $k$ is defined in (3.7).

We finally note that in the situation of Example 3.2 and Example 2.3 the constant $c$ is given by 0.05, 0.1 and 0.2, corresponding to the intervals $B_{A}, B_{B}$ and $B_{C}$ in (2.12) and the values for the critical point $k\left(\beta_{1}\right)$ are $k(1)=0.10768, k(0.5)=0.13860$ and $k(0.25)=0.16184$, respectively, which confirms the results given in Example 3.2.

\section{A remark concerning heteroscedasticity}

Several authors point out that many biological phenomena, where the Michaelis-Menten model is used, exhibit heteroscedasticity [see e.g. Currie (1982) or Dunn (1988).] Usually it is assumed that the conditional variance of $Y$ in $(2.2)$ is a function of the mean, i.e.

$$
\operatorname{Var}(Y \mid x)=v(g(x, a, b))
$$

where $g(x, a, b)=a x /(b+x)$ denotes the conditional expectation of $Y$ at $x$. Using results of Atkinson and Cook (1995) it can be shown that the Fisher information at the point $x$ in the Michaelis-Menten model (1.1) with heteroscedastic structure (4.1) is given by

$$
I_{v}(x, a, b)=\frac{g^{2}(x, a, b)}{a^{2}}\left(\begin{array}{cc}
1 & -\frac{a}{b+x} \\
-\frac{a}{b+x} & \frac{a^{2}}{(b+x)^{2}}
\end{array}\right)\left\{\frac{1}{v(g(x, a, b))}+\frac{1}{2}\left(\frac{v^{\prime}(g(x, a, b))}{v(g(x, a, b))}\right)^{2}\right\}
$$

and an analogous analysis as described in the homoscedastic case $(v \equiv 1)$ can be performed, where the structure of the standardized maximin $D$-optimal design depends on the assumed link function $v$. If

$$
M_{v}(\xi, a, b)=\int_{0}^{x_{0}} I_{v}(\xi, a, b) d \xi(x)
$$

denotes the information matrix of a design $\xi$ on $\left[0, x_{0}\right]$ and

$$
\xi_{a, b}^{*}=\arg \max _{\xi}\left|M_{v}(\xi, a, b)\right|
$$


is the locally $D$-optimal design, the standardized maximin $D$-optimal design maximizes

$$
\min \left\{\frac{\left|M_{v}(\xi, a, b)\right|}{\left|M_{v}\left(\xi_{a, b}^{*}, a, b\right)\right|} \mid(a, b) \in A \times B\right\},
$$

where $A, B \subset \mathbb{R}$ are given sets. For example Dunn (1988) postulated for receptor assays the quadratic relation $v(t)=t^{2}$, for which the solution of the optimization problem is particularly simple.

Theorem 4.1. For any set $A \times B$ the standardized maximin D-optimal design for the MichaelisMenten model (1.1) with heteroscedastic structure

$$
\operatorname{Var}(Y \mid x)=\left(\frac{a x}{b+x}\right)^{2}
$$

has equal masses at the points 0 and $x_{0}$. Moreover, this design is locally D-optimal for all parameter combinations of $(a, b)$.

Example 4.2. It might be of interest to compare the optimal designs under the assumption of homoscedasticity $(v(t) \equiv 1)$ and a quadratic link function $\left(v(t)=t^{2}\right)$. The standardized maximin $D$-optimal two point designs under the assumption of homoscedasticity are given in Example 2.3 and depend on the specified range for the non-linear parameter $b$. If a quadratic link function is assumed the optimal design does not depend on this range and has equal masses at the points 0 and $x_{0}$. This means that the structure of the design depends sensitively on the assumed link function for the variance.

\section{Appendix: proofs}

Proof of Theorem 2.2: It is easy to check that the optimal weights of a two point design must be equal [see e.g. Silvey (1980)]. If the support points are denoted by $z_{1}, z_{2} \in[0,1]$ we obtain for the Fisher information matrix of a design $\xi_{z_{1}, z_{2}}$ with equal weights at the points $z_{1}$ and $z_{2}$

$$
M(\xi, \beta)=\frac{1}{2} \sum_{i=1}^{2}\left(\begin{array}{cc}
\frac{z_{i}^{2}}{\left(z_{i}+\beta\right)^{2}} & -\frac{z_{i}^{2}}{\left(z_{i}+\beta\right)^{3}} \\
-\frac{z_{i}^{2}}{\left(z_{i}+\beta\right)^{3}} & \frac{z_{i}^{2}}{\left(z_{i}+\beta\right)^{4}}
\end{array}\right)
$$


and a straightforward calculation gives for the determinant

$$
|M(\xi, \beta)|=\frac{1}{4} \frac{z_{1}^{2} z_{2}^{2}\left(z_{2}-z_{1}\right)^{2}}{\left(z_{1}+\beta\right)^{4}\left(z_{1}+\beta\right)^{4}} .
$$

Therefore the standardized maximin $D$-optimality criterion reduces to

$$
\Phi\left(\xi_{z_{1}, z_{2}}\right)=\min \left\{16 \beta^{2}(1+\beta)^{6} \frac{z_{1}^{2} z_{2}^{2}\left(z_{2}-z_{1}\right)^{2}}{\left(z_{1}+\beta\right)^{4}\left(z_{2}+\beta\right)^{4}} \mid \beta \in\left[\beta_{0}, \beta_{1}\right]\right\}
$$

where we have used the representation for the determinant of the locally $D$-optimal design in (2.6). Now it is easy to see that $\Phi\left(\xi_{z_{1}, z_{2}}\right) \leq \Phi\left(\xi_{z_{1}, 1}\right)$ for all $z_{2} \in\left(z_{1}, 1\right]$ and consequently 1 is a support point of the best two point design. For $\xi_{z}=\xi_{z, 1}$ the criterion in (5.2) reduces to

$$
\Phi\left(\xi_{z}\right)=\min \left\{u(z, \beta) \mid \beta \in\left[\beta_{0}, \beta_{1}\right]\right\},
$$

where the function $u$ is defined by

$$
u(z, \beta)=16 \beta^{2}(1+\beta)^{2} \frac{z^{2}(1-z)^{2}}{(z+\beta)^{4}} .
$$

Recalling the definition of the point $z^{*}$ in $(2.11)$ we will prove below the following two assertions.

- For any fixed $z \in(0,1)$ the function $\beta \rightarrow \log u(z, \beta)$ is unimodal.

- If $\xi_{z^{*}}$ maximizes the function $\Phi$ in the class of all two point designs then

$$
u\left(z^{*}, \beta_{0}\right)=u\left(z^{*}, \beta_{1}\right)
$$

If these assertions have been established we obtain the representation

$$
\Phi\left(\xi_{z}\right)=\min \left\{u\left(z, \beta_{0}\right), u\left(z, \beta_{1}\right)\right\}
$$

for the criterion (5.3) from the unimodality of the function $\beta \rightarrow \log u(z, \beta)$, and (5.5) gives a condition on the optimal design $\xi_{z^{*}}$, which determines $z^{*}$ uniquely. Solving equation (5.5) with respect to $z^{*}$ it follows by a straightforward calculation

$$
z^{*}=z^{*}\left(\beta_{0}, \beta_{1}\right)=\frac{\beta_{1} \sqrt{\beta_{0}\left(1+\beta_{0}\right)}-\beta_{0} \sqrt{\beta_{1}\left(1+\beta_{1}\right)}}{\sqrt{\beta_{1}\left(1+\beta_{1}\right)}-\sqrt{\beta_{0}\left(1+\beta_{0}\right)}},
$$

which proves the assertion of Theorem 2.2. We complete the proof by showing the unimodality of the function $\beta \rightarrow \log u(z, \beta)$ and the identity (5.5). 
Proof of unimodality: This follows by a straightforward investigation of

$$
\frac{\partial}{\partial \beta} \log u(z, \beta)=\frac{1}{\beta}+\frac{1}{1+\beta}-\frac{2}{z+\beta}
$$

which has at most one zero for fixed $z$.

Proof of (5.5): Since the function $\beta \rightarrow \log u(z, \beta)$ has at most one maximum it follows that the standardized maximin $D$-optimal two point design $\xi_{z^{*}}$ can be found by maximizing the function $\Phi\left(\xi_{z}\right)$ defined in $(5.6)$ over the interval $(0,1)$. This maximization can be divided into three separate steps, namely the maximization over the sets

$$
\begin{aligned}
& \mathcal{M}_{<}:=\left\{z \in(0,1) \mid u\left(z, \beta_{0}\right)<u\left(z, \beta_{1}\right)\right\} \\
& \mathcal{M}_{>}:=\left\{z \in(0,1) \mid u\left(z, \beta_{0}\right)>u\left(z, \beta_{1}\right)\right\} \\
& \mathcal{M}_{=}:=\left\{z \in(0,1) \mid u\left(z, \beta_{0}\right)=u\left(z, \beta_{1}\right)\right\} .
\end{aligned}
$$

We will now demonstrate that the maximization over the first two sets yields a maximum at the boundary, which proves the assertion of (5.5). To this end consider the first set $\mathcal{M}_{<}$, the second case is treated exactly in the same way. A straightforward calculation shows

$$
\mathcal{M}_{<}=\left(0, z^{*}\right),
$$

where the point $z^{*}$ is defined in (5.7). Because for $z \in \mathcal{M}_{<}$we have $\Phi\left(\xi_{z}\right)=u\left(z, \beta_{0}\right)$ a straightforward maximization of $\Phi\left(\xi_{z}\right)$ over the set $\mathcal{M}_{<}$yields

$$
\tilde{z}=\frac{\beta_{0}}{1+2 \beta_{0}} \quad \text { and } \quad u\left(\tilde{z}, \beta_{0}\right)=1 .
$$

Now assume that $\tilde{z}$ is in the set $\mathcal{M}_{<}$then we must have $u\left(\tilde{z}, \beta_{0}\right)<u\left(\tilde{z}, \beta_{1}\right)$ and a tedious calculation shows that this inequality is equivalent to $\left(\beta_{0}-\beta_{1}\right)^{2}<0$. This contradiction shows that the maximum is not attained in the interior of the set $\mathcal{M}_{<}$and a similar argument for the set $\mathcal{M}_{>}$establishes the assertion (5.5) completing the proof of Theorem 2.2.

Proof of Lemma 3.1: It follows from Theorem 2.2 that $\mathcal{N}\left(\xi_{z^{*}}\right)=\left\{\beta_{0}, \beta_{1}\right\}$. If a prior $\pi$ puts weights $\alpha_{0}$ and $\alpha_{1}=\left(1-\alpha_{0}\right)$ at the points $\beta_{0}$ and $\beta_{1}$, respectively, then the inequality (2.10) can be rewritten as (note that $\alpha_{0}=1-\alpha_{1}$ )

$$
d\left(x, \alpha_{0}\right)=\sum_{i=0}^{1} \alpha_{i} f^{T}\left(x, \beta_{i}\right) M^{-1}\left(\xi^{*}, \beta_{i}\right) f\left(x, \beta_{i}\right) \leq 2 .
$$


If $\xi_{z^{*}}$ is in fact the (global) standardized maximin $D$-optimal design we must have equality for the support points of $\xi_{z^{*}}$. The equalities $d\left(1, \alpha_{0}\right)=d\left(z^{*}, \alpha_{0}\right)=2$ hold for all $\alpha_{0} \in(0,1)$ and therefore give no information regarding the weight. However at the point $x=z^{*}$ the function $d$ must have a local maximum, which yields the condition

$$
\left.\frac{\partial}{\partial x} d\left(x, \alpha_{0}\right)\right|_{x=z^{*}}=0
$$

Now a straightforward but very tedious calculation shows that this equation implies $\alpha_{0}=1 / 2$, which establishes the assertion of the Lemma.

Proof of Theorem 4.1: For $v(t)=t^{2}$ the Fisher information at the point $x$ in (4.2) reduces to

$$
I(x, a, b)=\frac{3}{a^{2}}\left(\begin{array}{cc}
1 & -\frac{a}{b+x} \\
-\frac{a}{b+x} & \frac{a^{2}}{(b+x)^{2}}
\end{array}\right)
$$

and it is easy to see that the best two point (locally) $D$-optimal design $\xi_{a, b}^{*}$ has equal masses at the points 0 and $x_{0}$ with information matrix

$$
M\left(\xi_{a, b}^{*}, a, b\right)=\frac{3}{2 a^{2}}\left(\begin{array}{cc}
2 & -a\left(\frac{1}{b}+\frac{1}{b+x_{0}}\right) \\
-a\left(\frac{1}{b}+\frac{1}{b+x_{0}}\right) & a^{2}\left(\frac{1}{b^{2}}+\frac{1}{\left(b+x_{0}\right)^{2}}\right)
\end{array}\right) .
$$

It is now straightforward to prove that the inequality

$$
\operatorname{tr}\left(I(x, a, b) M^{-1}\left(\xi_{a, b}^{*}, a, b\right)\right)=2 \frac{2 b x_{0} x^{2}+x_{0}^{2} x^{2}+b^{2} x^{2}+b^{2}\left(x_{0}-x\right)^{2}}{(b+x)^{2} x_{0}^{2}} \leq 2
$$

holds for all $x \in\left[0, x_{0}\right]$, which establishes the local $D$-optimality of the best two point design within the class of all designs. Since this design does not depend on $a$ and $b$ the assertion of Theorem 4.1 follows.

Acknowledgements: The authors would like to thank I. Gottschlich, who typed most parts of this paper with considerable technical expertise. Parts of this paper were written during a visit of the first author at Purdue University and this author would like to thank the Department 
of Statistics for their hospitality. The support of the Deutsche Forschungsgemeinschaft (DE 502/14-1 and SFB 475, Komplexitätsreduktion in multivariaten Datenstrukturen, Teilprojekt A2) is gratefully acknowledged.

\section{References}

A.C. Atkinson, R.D. Cook (1995). D-optimum designs for heteroscedastic linear models. J. Am. Stat. Assoc. 90, No.429, 204-212.

R.J.H. Beverton, S.J. Holt (1957). On the Dynamics of Exploited Fish Populations. London: Her Majesty's Stationary Office.

C.I. Bliss, A.T. James (1966). Fitting the rectangular hyperbola. Biometrics 22, 573-602.

E.P.J. Boer, D. Rasch, E.M.T. Hendrix (2000). Locally optimal designs in non-linear regression: A case study for the Michaelis-Menten function. Balakrishnan, N. (ed.) et al., Advances in Stochastic Simulation Methods, Selected papers at the 3rd St. Petersburg workshop on simulation, St. Petersburg, Russia, June 28 - July 3, 1998. Boston: Birkhäuser. Statistics for Industry and Technology, 177-188.

H. Chernoff (1953). Locally optimal designs for estimating parameters. Ann. Math. Statist. $24,586-602$.

A. Cornish-Browden (1979). Fundamentals of Enzyme Kinetics. Butterworth, London.

N.A.C. Cressie, D.D. Keightley (1979). The underlying structure of a direct linear plot with applications to the analysis of hormone-receptor interactions. Journal of Steroid Biochemistry 11, 1173-1180.

N.A.C. Cressie, D.D. Keightley (1981). Analysing data from hormone-receptor assays. Biometrics 37, 235-249.

D.J. Currie (1982). Estimating Michaelis-Menten parameters: Bias, variance and experimental design. Biometrics 38, 907-919.

H. Dette (1996). Lower bounds for efficiencies with applications. Research Developments in Probability and Statistics: Festschrift in honor of M.L. Puri's 65th birthday (eds. E. Brunner, M. Denker), 111-124. VSP Utrecht.

H. Dette (1997). Designing experiments with respect to standardized optimality criteria. J. Roy. Statist. Soc., Ser. B, 59, No.1, 97-110.

H. Dette, W.K. Wong (1999). E-optimal designs for the Michaelis-Menten model. Statistics \& Probability Letters 44, No.4, 405-408. 
R.G. Duggleby (1979). Experimental designs for estimating the kinetic parameters for enzymecatalysed reactions. Journal of Theoretical Biology 81, 671-684.

G. Dunn (1988). Optimal designs for drug, neurotransmitter and hormone receptor assays. Statistics in Medicine 7, 805-815.

N. Glick, A.D. Landman, B.D. Roufogalis (1979). Correcting Lineweaver-Burk calculations of $V$ and $K_{m}$. Trends in Biochemical Sciences 4, N82-N83.

L.M. Haines (1995). A geometric approach to optimal design for one-parameter nonlinear models. J. R. Stat. Soc., Ser. B 57, 575-598.

W.W. Hay, H.K. Meznarich, J.E. DiGiacomo, K. Hirst, G. Zerbe (1988). Effects of insulin and glucose concentration on glucose utilization in fetal sheep. Pediatric Research 23, 381-387.

L.A. Imhof (2001). Maximin designs for exponential growth models and heteroscedastic polynomial models. Ann. Statist. 29, No.2, 561-576.

S. Johansen (1984). Functional Relations, Random Coefficients and Nonlinear Regression, with Application to Kinetic Data. Lecture Notes in Statistics, 22. Springer, N.Y.

C.H. Müller (1995). Maximin efficient designs for estimating nonlinear aspects in linear models. J. Statist. Plann. Inference 44, No.1, 117-132.

F. Pukelsheim (1993). Optimal design of experiments. Wiley, N.Y.

J.G.W. Raaijmaakers (1987). Statistical analysis of the Michaelis-Menten equation. Biometrics 43, No.4, 793-803.

D. Rasch (1990). Optimum experimental design in nonlinear regression. Commun. Statist., Theor. Meth. 19, No.12, 4789-4806.

S.D. Silvey (1980). Optimal design. Chapman and Hall, London.

D. Song, W.K. Wong (1998). Optimal two point designs for the Michaelis-Menten model with heteroscedastic errors. Commun. Statist., Theory Meth. 27, No.6, 1503-1516.

W.K. Wong (1992). A unified approach to the construction of minimax designs. Biometrika 79, No.3, 611-619.

J.A. Zivin, D.R. Waud (1982). How to analyze binding, enzyme and uptake data: the simplest case, a single phase. Life Sciences 30, 1407-1422. 